\title{
CORRIGENDUM
}

\section{Corrigendum: RPN2 gene confers docetaxel resistance in breast cancer}

Kimi Honma, Kyoko Iwao-Koizumi, Fumitaka Takeshita, Yusuke Yamamoto, Teruhiko Yoshida, Kazuto Nishio, Shunji Nagahara, Kikuya Kato \& Takahiro Ochiya

Nat. Med. 14, 939-948 (2008); published online 17 August 2008; corrected after print 7 October 2008

In the version of this article initially published, the protein ribophorin II was incorrectly referred to as ribopholin II. The error has been corrected in the HTML and PDF versions of the article. 